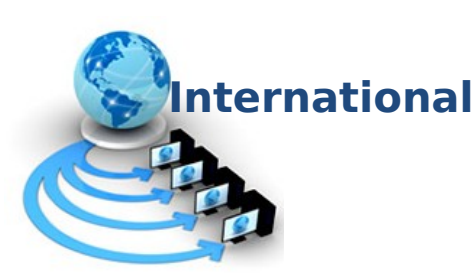

Volume 11, No. 4, July-August 2020

Journal of Advanced Research in Computer Science

RESEARCH PAPER

Available Online at www.ijarcs.info

\title{
AN ADVANCED FACE DETECTION AND RECOGNITION
}

\author{
Anirban Chakraborty \\ Department Of Artificial Intelligence \\ Lovely Professional University \\ Phagwara, Punjab, India
}

\author{
Sai Sowjanya Kallempudi \\ Department Of Artificial Intelligence \\ Lovely Professional University \\ Phagwara, Punjab, India
}

\begin{abstract}
Image or video Face Identification is a popular subject for research in biometrics. Most public places usually have video capture surveillance cameras and these devices have an important safety benefit. The identification of the face has played a significant role in the monitoring system since the entity does not need assistance. It is widely recognized all over the world. Uniqueness and Validation are the main benefits of facial recognition over other biometrics. Since, Human face is a highly variable and dynamic subject, the detection of the face in computer vision is a difficult problem. Precision and Recognition speed are a big problem in this area. The purpose of this paper is to test various facial detection and recognition approaches, and provide a full solution for facial detection and recognition in an image-based manner with a higher degree of accuracy and an improved response time. This suggests a solution focused on experiments carried out on different facial rich datasets with respect to topics, images, attitudes, ethnicity and color.
\end{abstract}

Keywords: Machine Learning; Image; Processing; Neural Networks; Artificial Intelligence; Deep Learning

\section{INTRODUCTION}

There has been a great deal of research over the last decade on identifying and recognizing people ${ }^{[14]}$, as this is the easiest way to recognize people ${ }^{[16]}$ and human cooperation is not necessary ${ }^{[15]}$ over order for biometrics to become a hot subject. Because several tracking ${ }^{[6,7,8,12,13]}$ and surveillance ${ }^{[8,9,10,11]}$ approaches have been implemented, they are called a Landmark. While the methods are used in past years for the same reason many times separately with a small number of data sets, it is baseless who gives a complete assessment of the output of these methods by checking them on hard datasets in Facial Databse ${ }^{[1,2,3,4,5]}$. A first milestone for video-based facial detection and monitoring recognition was developed in the current paper for the method assessment. Figure 1 displays the description of the new framework.

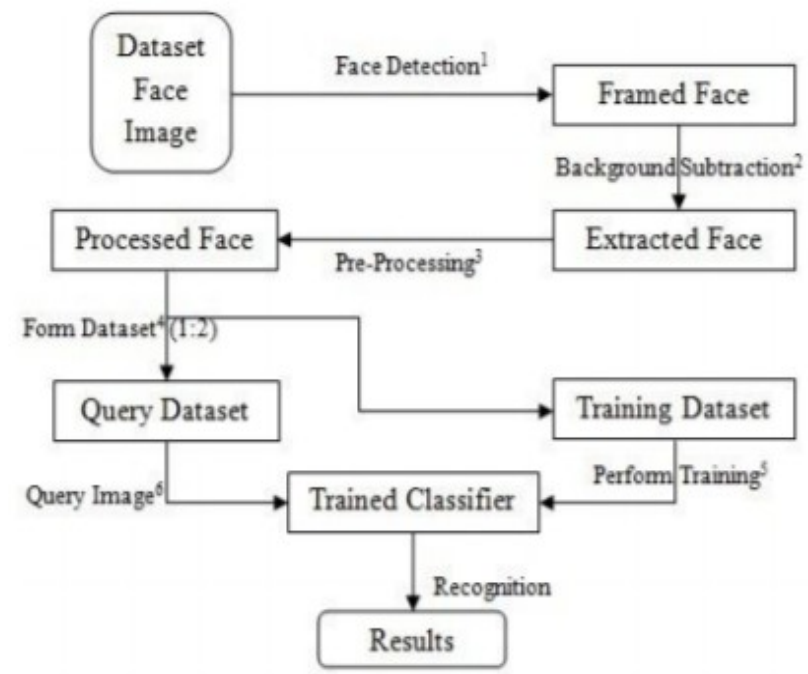

Fig. 1 System's overview.
Section III of the paper addresses facial recognition methods focused on the findings of Section II. Facial detection clinic AdaBoost ${ }^{[6]}$ is used with Hair ${ }^{[7]}$ and Local binary Pattern(LBP) ${ }^{[8]}$ feature, while the Facial Detection Histogram (HOG) $)^{[14]}$ with a Support Vector Machine(SVM) ${ }^{[12]}$ is used for facial detection. A modern imaging system that offers a range of functions uses AdaBoost enhancement algorithm ${ }^{[6]}$ to minimize increased classification and degeneration technique to give robust and quick intervention but only basic rectangular hair-like features $^{[7]}$, which provide many benefits, including any kind of ad hoc data. Hairlike features are determined using a modern image representation. Implementing a system has too many features, so the feature must be limited to only a few essential dataset that can be incorporated using the Adaboost algorithm ${ }^{[6]}$. The original LBP ${ }^{[8]}$ operator marks a picture pixel, use the binary number and the central pixel value to thrash every $3 / 3$ pixel in the neighborhood. Every image mask can compose the micro-setting patterns that can effectively be identified by the LBP operator ${ }^{[8]}$. We separated face pictures into n tiny regions $\boldsymbol{T} \mathbf{0}, \boldsymbol{T} \mathbf{1}, \ldots, \boldsymbol{T N}$ for description of the facial form. The histogram $\mathrm{LBP}^{[8]}$ from the different sub-regions is then related to the following specification, in a spatially improved histogram:

$H i, j=\Sigma x, y I(f l(x, y)=i) I((x, y) \epsilon T j)$

where $\mathbf{i}=\mathbf{0}$ to $\mathrm{L}-\mathbf{1} ; \mathbf{j}=\mathbf{0}$ to $\mathbf{N}-1$.

The extracted feature Histogram describes the local texture and global shape of face images. 



Threshold

\section{$(10000011)^{2}=131$}

Fig. 2 LBP calculation.

\section{FACE DETECTION}

Many Literature Surveys are done before performing the experiment. These are as follows:

i. Hannes Schulz - Sven Behnke proposed that Hierarchical neural networks have a long history for object recognition. Throughout recent years, new methods have been introduced throughout order to slowly acquire a hierarchy of features from unlabeled inputs. Together with developments from parallel machines, these deep learning methods made it possible, for the profundity and input scale of problems not feasible before, to be effectively tackled. In this essay, we provide the readers with fundamental concepts of profound thinking, analyze chosen approaches in depth and provide examples of implementation from computer vision and speech recognition.

ii. Birgit Pfarrkirchner Christina Gsaxner stated that The segmentation sector is a significant field in the production of diagnostic photographs and the foundation for more detailed studies on computed tomography (CT), MRI, X-ray, ultrasound (US), or nuclear pictures. The image is divided into different connected areas which correspond to certain types of tissue by segmentation. Another common goal is to delineate unhealthy and stable tissues. The tumor and pathological lesion detection and its magnitude to determine clinical plans and effects are a common source in medicine. For the preparation of specific treatment procedures, for instance in radiation, segmentation is included in the clinical routine.

iii. Suvajit Dutta Bonthala C S Manideep stated that Diabetes (DM) is a metabolic disorder that is due to the high content of the body's blood sugar. Diabetic retinopathy (DR) also causes major sight impairment. Diabetes also contributes to eye deterioration over time. Increased blood pressure, fluid decrease, exudates, bleeding and micro anneurysms may cause the symptoms in the retinal region. Images are the essential tool for successful patient treatment in modern medical science. While, interpretation of modern medical terminology is still difficult. The Deep neural networks computer vision will accurately train a robot and the accuracy level is also better than other neural networking models. After evaluating the models with a qualified CCU neural network the suggested models are programmed with three types: NN rear propagation; Deep Neuro Network (DN Network); and Convolutionary Neural Network (CNN) after reviewing models. The Deep Learning models will quantify the characteristics of blood vessels, fluid rises, exudates, blood cell blood cells and microaneurysms of different classes. The algorithm measures the weight that brings the patient's eye frequency.

iv. Sameer Khurana1, Reda Rawi2, Khalid Kunji3, Gwo-Yu Chuang2, Halima Bensmail3 and Raghvendra Mall3, said that Throughout pharmaceutical research and production efficiency, protein solubility plays a vital role.The degree of its solubility will, and is determined by its sequence, constitute the nature of a given protein's operation. Therefore, it is important to develop new, highly accurate protein solubility predictors based on silicon sequence. In this study, DeepSol is a novel Protein Solubility Predictor focused on Deep Learning. A neural network that leverages k-mer structure and additional sequent and structural functions derived from the protein sequence is the cornerstone of our architecture.

v. Francis Quintal Lauzon stated that Deep learning allows multiple layers of description to be dynamically modelled of the underlying data distribution. A special program called stacked denoising autoencoders is examined in this research. This kind of view, combined with an SVM, improves MNIST classification errors over the standard deep learning strategy, when a logistic regression layer with denoising autoencoders is applied to the stack.An easy way to comply with the conference paper formatting requirements is to use this document as a template and simply type your text into it.

vi. Deep neural networks (DNNs) typically have millions, possibly billions, of parameters / weights which are very costly both for storage and measurement. This lead to a broad range of work by using regulators that reduce the sophistication of the neural network. The parameter sharing / bonding, in which certain sets of weights have to share a common values, is another popular approach for managing DNN complexities. Some forms of weight sharing are hard to express some variances, with the change invariance of convolutionary layers being a notable example.

\section{FACE DETECTION}

For face recognition, HOG ${ }^{[13]}$ technologies have been used for the SVM classifier ${ }^{[12]}$. HOG ${ }^{[13]}$ passes wavelets and smoother rates before measuring harm to the gradient, the findings emphasize that most of the knowledge accessible on sudden edges on fine scales is mistaken to blur. This is to reduce the spatial sensitivity. Gradients in the current pyramid layer should be measured at the finest possible scale and a clear local contrast normalization for successful results is important. In comparison, $\mathrm{SVM}^{[12]}$ is developed in order to address a traditional two-class problem returning a binary type in the entity class. We are formulating the question in an environment where there are various variables in the training 
of our SVM ${ }^{[12]}$ algorithm. The experiment results are given below for the above methods.

Table 1: Face detection results summery

\begin{tabular}{|c|c|c|c|}
\hline \multirow{2}{*}{ Dataset } & \multicolumn{3}{|c|}{ Detection } \\
\cline { 2 - 4 } & \multicolumn{2}{|c|}{ Adaboost } & SVM \\
\cline { 2 - 4 } & Haar & LBP & HOG \\
\hline \multirow{1}{*}{$\mid$} & $99.31 \%$ & $95.22 \%$ & $92.68 \%$ \\
\hline$|2|$ & $98.33 \%$ & $98.96 \%$ & $94.10 \%$ \\
\hline$|3|$ & $98.31 \%$ & $69.83 \%$ & $87.89 \%$ \\
\hline$|4|$ & $96.94 \%$ & $94.16 \%$ & $90.58 \%$ \\
\hline$|5|$ & $90.65 \%$ & $88.31 \%$ & $89.19 \%$ \\
\hline Mean & $\mathbf{9 6 . 7 0} \%$ & $89.30 \%$ & $\mathbf{9 0 . 8 8} \%$ \\
\hline
\end{tabular}

Moreover system is been tested on datasets ${ }^{[1,2,3,4,5]}$ and based on above demonstrated system, results are demonstrated below:



Fig. 3 Face detection.

Two additional actions performed in the pre-processing stage to enhance the recognition results were used to reduce pose variation and illumination on extracted face.

1. The eye detection was used to remove head turn, tilt, slant and face location, as shown in Figure 4.

2. The histogram equalization was done.

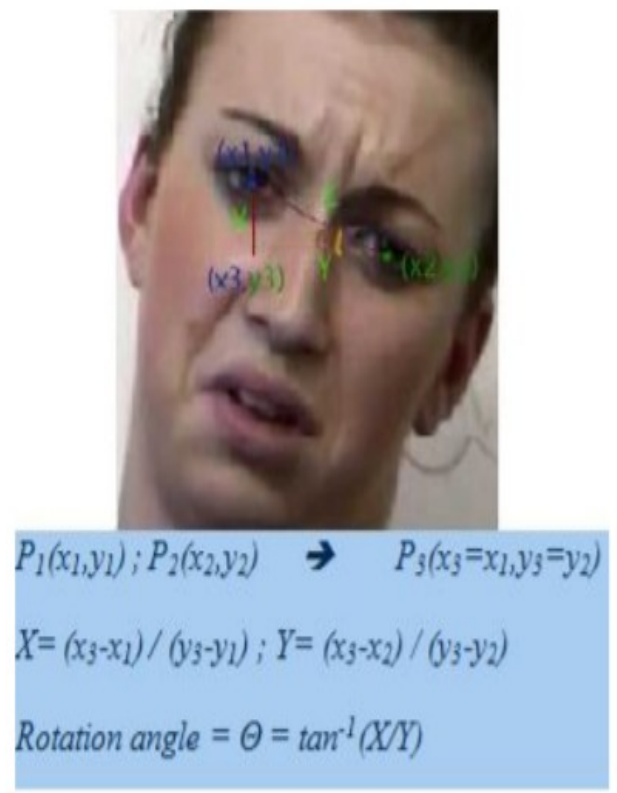

Fig. 4 Face rotation.

\section{FACE RECOGNITION}

The characteristics ${ }^{[9]}$ seen as a concern with $2 \mathrm{D}$ facial recognition, are mostly upright and broad. This is why 3-D facial knowledge is not required, which greatly reduces the difficulty. This translates face frames into a variety of simple roles which are the key components of facial frames and finds ways to reflect the data more effectively. It is particularly beneficial as the measurement effort is popular. The primary aim is to reduce the amount of characteristics before identification to a more controlled amount, because face is defined by a widespread number of pixel values. A regular arrangement of pixel values creating a pattern is used for all new measurements. Fisherfaces ${ }^{[10]}$ is the name of the vector varieties achieved by using Fisher's conditional discriminant. $\mathrm{LBP}^{[8]}$ is a collection of discrete pixel size measurements between the pixel core and the 8 adjacent pixels.

\section{$\operatorname{LBP}(x a, y a)=7 \Sigma n=0$ s $(i m-i a) 2 n$}

Where,

$i a$ corresponds to the value of the center pixel $(x a, y a)$, im corresponds to the value of eight surrounding pixels,

function $\mathrm{f}(\mathrm{x})$ is defined as:

$$
\begin{array}{r}
f(x)=1 \text { if } x>=0 \\
0 \text { if } x<0
\end{array}
$$

Filters from Gabor ${ }^{[1]}$ will manipulate influential visual properties including spatial position, selectiveness of orientation and spatial frequency properties. Gabor ${ }^{[11]}$ characteristics are different to transformations as lighting, pose and voice while transformation of Gabor ${ }^{[1]}$ is not expressively built for face recognition in the light of such devastating potential and great performance. Instead of being learned from face training information, the transformation formula is predefined. In addition, $\mathbf{P C A}{ }^{[10]}$ and Classifier $\mathbf{L D A}^{[9]}$ consider global features, while $\mathrm{LBP}^{[8]}$ and the Classifier Gabor consider local features. 
Table 2: Face recognition results summery

\begin{tabular}{|c|c|c|c|c|}
\hline \multirow{2}{*}{ Dataset } & \multicolumn{4}{|c|}{ Recognition } \\
\cline { 2 - 5 } & PCA & LDA & LBP & Gabor \\
\hline$[1]$ & $72.10 \%$ & $79.39 \%$ & $85.93 \%$ & $93.49 \%$ \\
\hline$[2]$ & $69.87 \%$ & $76.61 \%$ & $80.47 \%$ & $89.76 \%$ \\
\hline$[3 \mid$ & $70.95 \%$ & $78.34 \%$ & $84.14 \%$ & $92.68 \%$ \\
\hline$[4 \mid$ & $74.79 \%$ & $81.93 \%$ & $86.45 \%$ & $96.91 \%$ \\
\hline$[5 \mid$ & $68.04 \%$ & $73.21 \%$ & $77.69 \%$ & $88.93 \%$ \\
\hline Mean & $71.15 \%$ & $77.90 \%$ & $82.94 \%$ & $92.35 \%$ \\
\hline
\end{tabular}

\section{DATASeTS}

In the experiment, five datasets were included.

- The data set ${ }^{[1]}$ includes a smooth green backdrop facial collection; there is no head and light variance, but there are slight eye incline, slant, location and important shifts in the gestures.

- In dataset ${ }^{[2]}$, the selection of the face with red curtains backdrop shadows are attributed to variability when the body shifts in front of it with slight adjustments in the head turning, tilting and slanting broad head size variance, some variability in voice, transition in the direction of the face and picture illumination changes with the subject shifting in front of the body.

- In dataset [3], complicated context facial collection; broad differences in the direction of the eye; small adjustments in eye shift, tilting, slant and expression; certain face angle transfer and major light variance attribute to the artificial light moment item were used.

- In dataset [4], smooth context facial collection; minor difference in head scale; large variation in head tilt, inclination, slim and big variation in expressiveness; limited frontal transmission and light variability were shown.

- In dataset [5], face with a steady backdrop with small shifts in head scale and light; fantastic adjustments in shape, shift, slant, posture and the face location were identified.

\section{CONCLUSION}

In current work we developed the system to evaluate the face detection and recognition methods which are
Table 3: Face database summary

\begin{tabular}{|c|c|c|c|c|c|}
\hline Data Set & Sub-Division & Images & Resolution & Individuals & Image Individual \\
\hline \multirow{4}{*}{1} & Face 94 & 3078 & 180200 & 153 & 20 \\
\hline & Facess & 1440 & 180200 & 12 & 20 \\
\hline & Facey & 3016 & $196^{\circ} 19 \%$ & 152 & 20 \\
\hline & Grimace & 360 & 180200 & 18 & 20 \\
\hline B & Pain Expressions & 599 & 7204576 & 23 & 26 \\
\hline
\end{tabular}

A:Face Recognition Data, University of Essex

B: Psychological Image Collection at Stirling (PICS) considered to be a bench mark.

While other approaches are rather unpredictable, but based on the average outcomes, five datasets were used for this reason, and the average experimental findings are analyzed. Table 1 and Table 2 are the experimental findings for facial recognition and identification techniques, while the summery data sets are given in Table 3 respectively. Hairlike $^{[7]}$ features are recorded fairly effectively in the current Haar-like framework, but there is more mis-detection than $\mathrm{LBP}^{[8]}$, which can be seen as a potential research in tracking, minimizing false detection of hair-look ${ }^{[7]}$ characteristics.

\section{ACKNOWLEDGEMENT}

We, the authors are very much thankful to Mrs. Rita Biswas for funding our research.

\section{REFERENCES}

[1] Kaur, Bhupinder \& Chakraborty, Anirban. (2020). Fac spoof identification strategies and implementation: outcomes and insight. 10.13140/RG.2.2.24885.70886.

[2] Sun, Yi, Xiaogang Wang, and Xiaoou Tang. "Hybrid Deep Learning for Face Verification." IEEE Transactions on Pattern Analysis and Machine Intelligence 38.10 (2016): 1997-2009.

[3] Hu, Guosheng, et al. "When face recognition meets with deep learning: an evaluation of convolutional neural networks for face recognition." Proceedings of the IEEE International Conference on Computer Vision Workshops. 2015.

[4] Goodfellow, Ian, Yoshua Bengio, and Aaron Courville. Deep learning. MIT Press, 2016.

[5] Zhang, Tong, et al. "A deep neural network driven feature learning method for multi-view facial expression recognition." IEEE Trans. Multimed 99 (2016): 1 .

[6] Lawrence, Steve, et al. "Face recognition: A convolutional neural-network approach." IEEE transactions on neural networks 8.1 (1997): 98-113.

[7] Srivastava, Nitish, et al. "Dropout: a simple way to prevent neural networks from overfitting." Journal of Machine Learning Research 15.1 (2014): 1929-1958.

[8] Kanade, Takeo. "Picture processing system by computer complex and recognition of human faces" Doctoral dissertation, Kyoto University 3952 (1973): 83-97.

[9] Brunelli, Roberto, and Tomaso Poggio. "Face recognition: Features versus templates." IEEE transactions on pattern analysis and machine intelligence 15.10 (1993): 1042-1052. 
[10] Cox, Ingemar J., Joumana Ghosn, and Peter N. Yianilos. "Feature-based face recognition using mixturedistance." Computer Vision and Pattern Recognition, 1996. Proceedings CVPR'96, 1996 IEEE Computer Society Conference on. IEEE, 1996.

[11] Ahonen, Timo, et al. "Recognition of blurred faces using local phase quantization." Pattern Recognition, 2008. ICPR 2008. 19th International Conference on. IEEE, 2008.

[12] Kohonen, Teuvo, and Self-Organizing Maps. "vol. 30." Berlin, Heidelberg, New York: Springer 1997 (1995): 2001. Wiskott, Laurenz, et al. "Face recognition and gender determination." (1995): 92-97.

[13] Maas, Andrew L., Awni Y. Hannun, and Andrew Y. Ng. "Rectifier nonlinearities improve neural network acoustic models." Proc. ICML. Vol. 30. No. 1. 2013.

[14] Chan, Chi Ho, et al. "Multiscale local phase quantization for robust component-based face recognition using kernel fusion of multiple descriptors." IEEE Transactions on Pattern Analysis and Machine Intelligence 35.5 (2013): 1164-1177.

[15] S. Moore and R. Bowden, Local binary patterns for multi-view facial expression recognition. Computer Vision and Image Understanding, 2011, pp. 541-558.
[16] Ahonen, Timo, Abdenour Hadid, and Matti Pietikäinen. "Face recognition with local binary patterns." Computer vision-eccv 2004 (2004): 469481.

[17] M. Dahmane and J. Meunier, Emotion recognition using dynamic gridbased $\mathrm{HoG}$ features. Proc. IEEE International Conference on Automatic Face and Gesture Recognition and Workshops, 2011, pp. 884888.

[18] Turk, Matthew, and Alex Pentland. "Eigenfaces for recognition." Journal of cognitive neuroscience 3.1 (1991): 71-86.

[19] P. Ekman, W. V. Friesen, and J. C. Hager, Facial Action Coding System: The Manual on CD ROM. Research Nexus Division of Network Information Research

[20]D. G. Lowe, "Distinctive Image Features from ScaleInvariant Keypoints," International Journal of Computer Vision, vol. 60, no. 2, pp. 91-110, Nov. 2004.

[21]D. G. Lowe, "Object Recognition from local scaleinvariant keypoints," In proc. of International Conference on Computer Vision, pp. 1150-1157, 1999.

\section{Corresponding Author,}

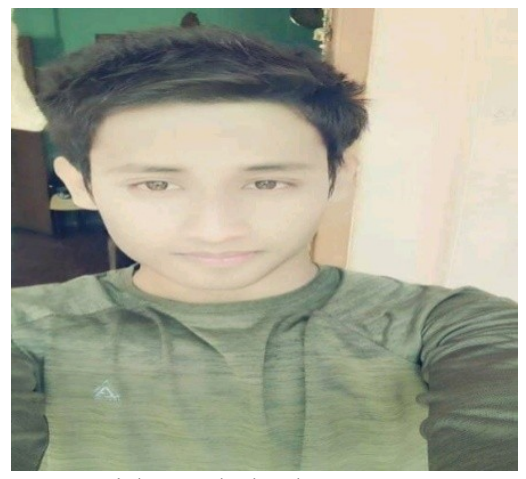

Mr. Anirban Chakraborty

Miss. Sai Sowjanya Kallempudi

Co-Author

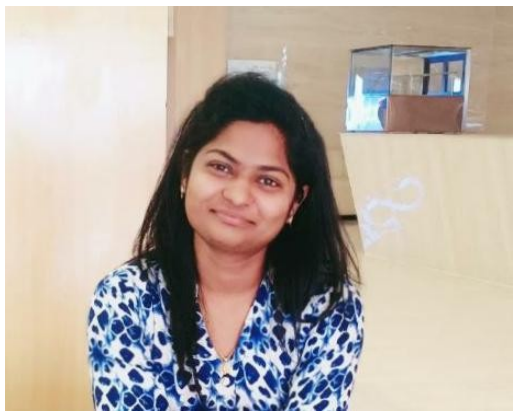

